\title{
Pulmonary Sequestration as an Incidental Finding of Pediatric Abdominal Ultrasound - Two Cases
}

\author{
Jovan Lovrenski, M.D., Ph.D.
}

Radiology Department, Institute for Children and Adolescents Health Care of Vojvodina, Faculty of Medicine, University of Novi Sad, Hajduk Veljkova 3, Novi Sad 21000, Serbia.

Received 31 July 2018 • Revised 25 September 2018 • Accepted 1 October 2018 • Published online 8 November 2018

\section{Abstract:}

Abdominal ultrasound (US) is a commonly indicated examination in pediatrics. However, lung bases are often not thoroughly examined. Pulmonary sequestration is mostly detected prenatally, but also postnatally - incidentally or with the appearance of recurrent pulmonary infections. It can be detected by US, yet our research has not found a single reported case where sequestration was incidentally detected by US, without any previous knowledge of its existence. Here, we report on 2 such cases of pulmonary sequestrations detected during routine pediatric abdominal US. Tubular anechogenic structure above the left hemidiaphragm should bring attention to the potential diagnosis of pulmonary sequestration.

Keywords: abdomen, lung, pediatrics, pulmonary sequestration, ultrasound

Contact: Assoc. Prof. Jovan Lovrenski, M.D., Ph.D.

Radiology Department, Institute for Children and Adolescents Health Care of Vojvodina, Faculty of Medicine, University of Novi Sad, Hajduk Veljkova 3, Novi Sad 21000, Serbia.

E-mail address: JOVAN.LOVRENSKI@mf.uns.ac.rs
J Health Sci Med Res 2019;37(1):61-66 doi: 10.31584 /jhsmr.032 www.jhsmr.org 


\section{Introduction}

Pulmonary sequestration is a congenital anomaly that accounts for up to $6.4 \%$ of all bronchopulmonary foregut malformations. ${ }^{1}$ It is a non-functioning lung tissue mass that does not communicate with the tracheobronchial tree, with an arterial blood supply coming directly from the systemic circulation, most commonly the aorta. ${ }^{2}$

Demonstration of the anomalous arterial supply is crucial for the diagnosis. It can be achieved prenatally, using mostly color Doppler ultrasound (CDUS) and 3D power Doppler US, but also via fetal magnetic resonance imaging ( $\mathrm{fMRI}$ ), as well as postnatally by using computed tomography (CT), CDUS or MRI. ${ }^{3-8}$

We report on 2 cases in which pulmonary sequestration was postnatally diagnosed by US incidentally, while performing an abdominal US, without its antenatal or postnatal diagnosis. During an extensive search through available data bases, we have only found reports of US of pulmonary sequestration when the postnatal US exam was performed after its prenatal diagnosis, or after the postnatally raised suspicion of sequestrum based on the chest X-ray (CXR). ${ }^{5,9,10}$ Therefore, in all these cases, the examiner knew exactly what to look for when performing US. Conversely, in our cases, the US was the first diagnostic modality demonstrating the finding of pulmonary sequestration, without any hint of its existence.

The aim of this report is to emphasize the importance of the transabdominal approach to the lung bases when performing abdominal US in children, and to identify which US finding might, when observed, be suggestive of pulmonary sequestration.

\section{Case 1}

A 1-month-old girl presented to the Pediatric Emergency Department, and was reported to have been vomiting for 2 days. She was a full-term infant, born by cesarean delivery, with an Apgar score 9, 10. She was immediately sent to the Radiology Department for an abdominal US by her pediatrician.

During the US examination (Acuson S2000, Siemens, Erlangen, Germany) of the spleen, a trans-splenic approach by linear probe of $7 \mathrm{MHz}$ enabled visualization of the left pulmonary base consolidation with tubular anechogenic structure within it (Figure 1). CDUS showed a blood flow within the described tubule, stemming from a systemic arterial supply from the aorta just above the diaphragm, which is consistent with pulmonary sequestration (Figure 2). Contrast-enhanced CT (arterial phase only) confirmed the US finding (Figure 3). It also showed the venous drainage of sequestration into the hemiazygos vein.

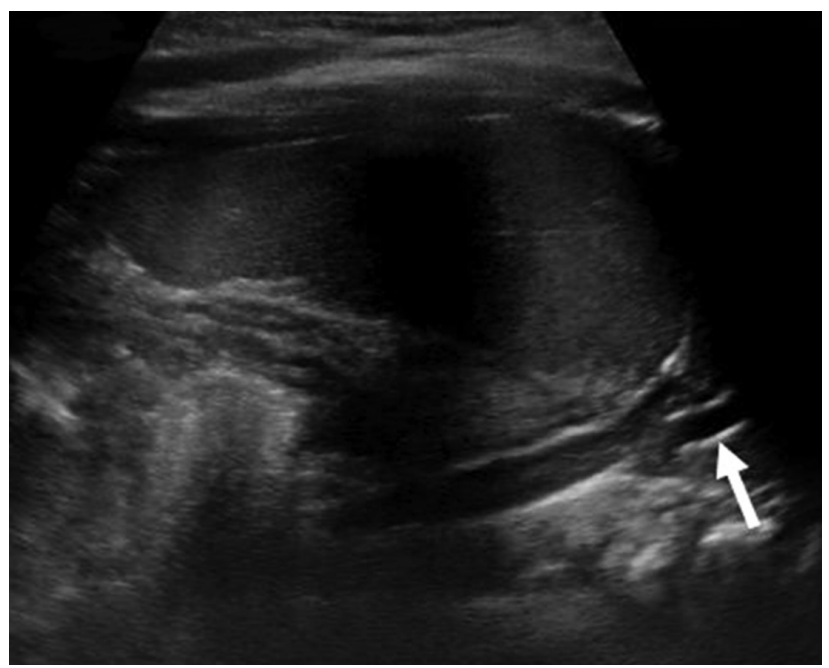

Figure 1 Anechogenic tubular structure (arrow) was seen trans-splenically (longitudinal section) within the visualized part of the consolidated left lung base, on a routine abdominal ultrasound in a 1-month-old girl with reported vomiting. 


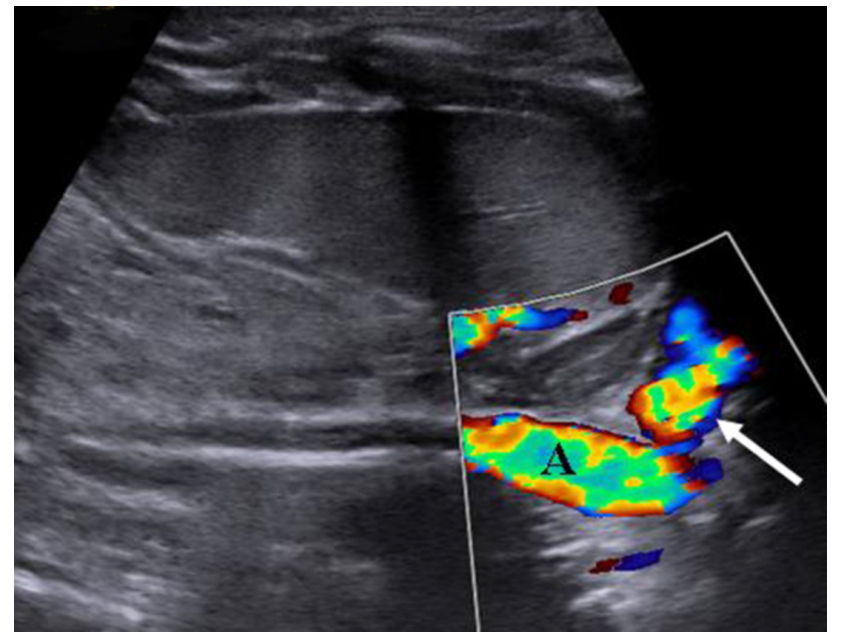

a

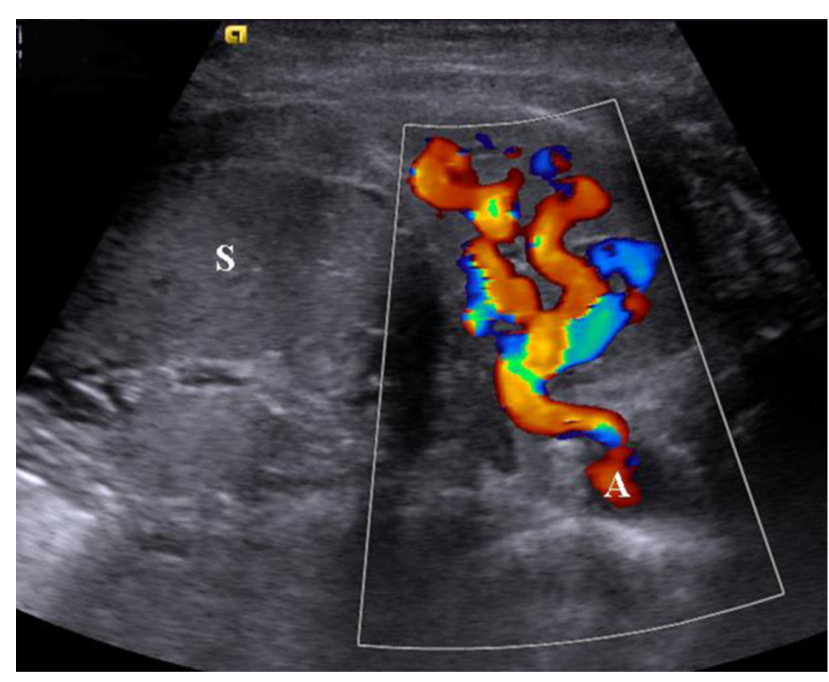

b

Figure 2 Color Doppler ultrasound in the same girl as in Figure 1 shows a systemic arterial supply (arrow) from the descending aorta $(A)$ of the left lung base, visualized trans-splenically, on the longitudinal (a) and transverse (b) section, consistent with pulmonary sequestration. S - spleen.

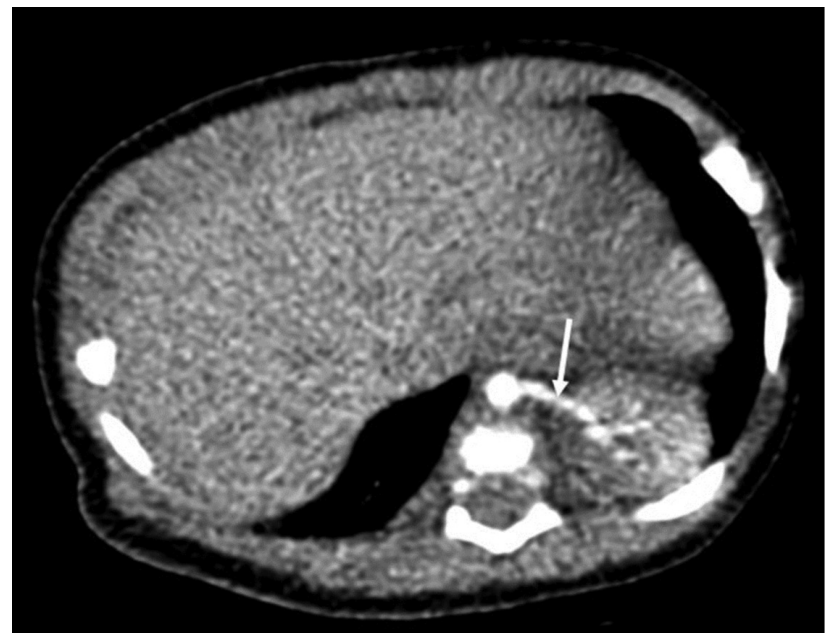

Figure 3 Axial contrast-enhanced computed tomography scan in the arterial phase shows a systemic arterial supply (arrow) of pulmonary sequestration, positioned within the left lung base in the same girl as in figures 1 and 2 .

There have not been any sequestration related complications in this child, and she is still followed up periodically by US.

\section{Case 2}

A 17.5-year-old girl presented to the Pediatric Emergency Department with a fever of unknown origin, malaise, and pain under the left costal arch. At admission, C-reactive protein (CRP) was elevated - $157 \mathrm{mg}$ /, as well as erythrocyte sedimentation rate (ESR) - $100 \mathrm{~mm} / \mathrm{hr}$, while all the other laboratory tests were negative. Apart from tenderness in the left upper part of the abdomen, physical findings were unremarkable. She had multiple hospitalizations for idiopathic juvenile arthritis, the last one having been 2 years prior, and had had no health issues since. 
Several abdominal US examinations were performed over the past six years. Abdominal ultrasound (Acuson S2000, Siemens, Erlangen, Germany) on the day of admission showed only the splenomegaly. Transsplenically, however, a consolidation in the left lung base was observed using a convex probe of $5 \mathrm{MHz}$, and within it a tubular, branching, anechogenic structure with an arterial flow on CDUS, communicating with the aorta just below the diaphragm. The finding was unequivocally consistent with pulmonary sequestration (Figure 4). Parenteral antibiotic therapy was started in conjunction with antipyretic therapy. From the third day of admission, the patient showed significant clinical improvement, with disappearance of fever and left upper abdominal quadrant pain. CRP and ESR also decreased to $24 \mathrm{mg} /$ and $60 \mathrm{~mm} / \mathrm{hr}$, respectively. US finding of sequestration was confirmed by contrast-enhanced CT, with only the arterial phase performed. A venous drainage of sequestration was into the azygos vein.

The last follow-up in this girl was at the age of 18 , without any disease-related complications. She is planned to undergo a surgical procedure at the Clinic for Thoracic Surgery (adult's clinic, since she is no longer a pediatric patient).

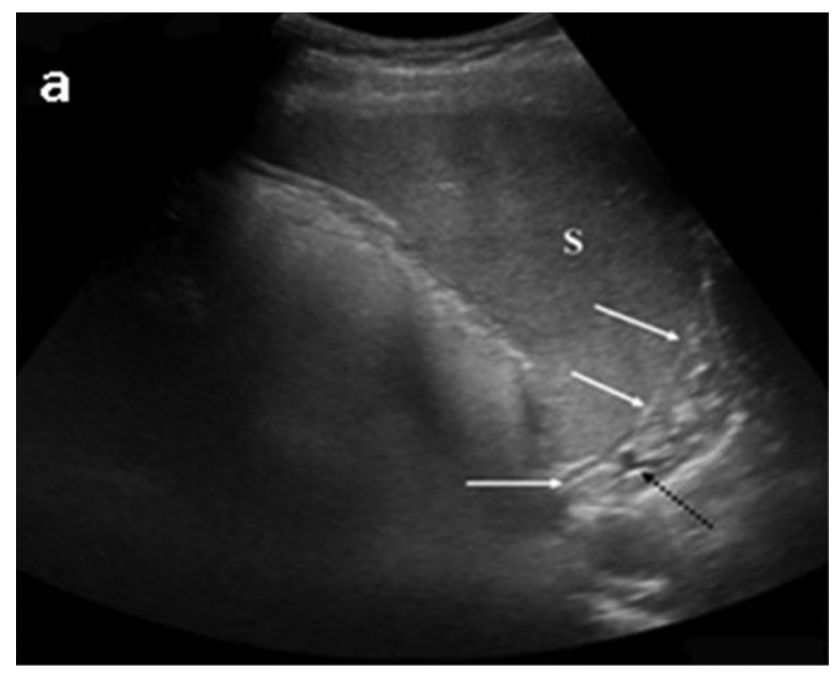

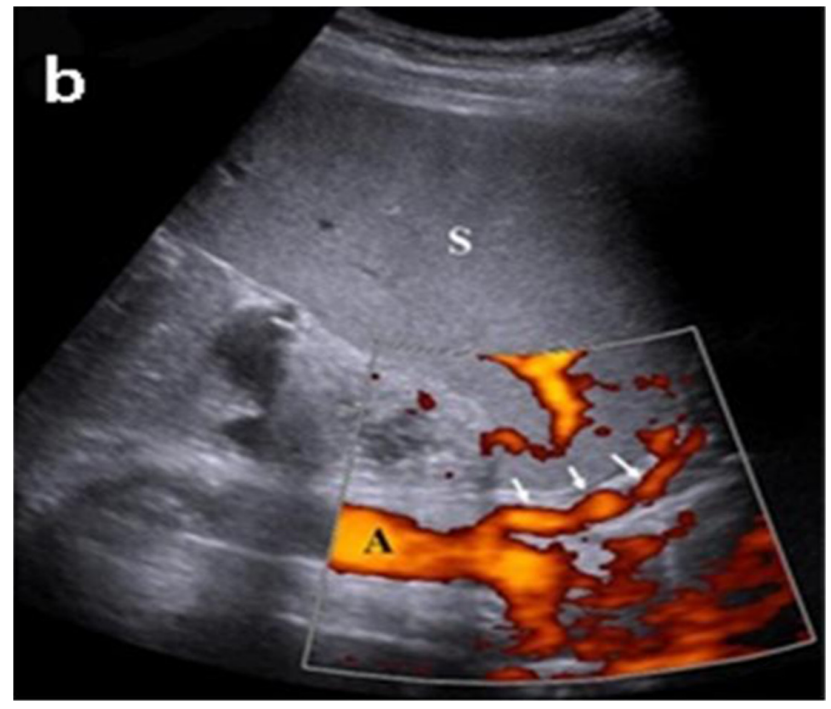

b

Figure 4 A 17.5-year-old girl was admitted to the hospital due to a fever of unknown origin. Trans-splenic ultrasound approach (longitudinal section) to the left lung base (a) revealed a consolidation (white arrows) with a branching anechogenic structure (black dashed arrow).

(b) On power Doppler this consolidation corresponded to pulmonary sequestration, with a clearly detected systemic arterial supply from descending aorta (A) (aortic branch "feeding" the consolidation is marked with white arrows). S - spleen.

\section{Discussion}

Abdominal US is one of the most commonly indicated radiological diagnostic procedures in the pediatric population, if not the most frequent one. However, it would appear the lung bases are still not the primary focus of pediatric radiologists when performing routine abdominal US, even though they are easily accessible due to the mostly small amount of subcutaneous fat tissue, resulting in better resolution of US images. Our goal was 
to draw attention to the possibility of US diagnosis of sequestration, even incidentally, when the radiologist is not aware of the potential lung base pathology. While we did find articles describing postnatal US diagnosis of pulmonary sequestration, all of these addressed a "focused" US examination, as sequestration has been an incidental finding on prenatal US, or postnatal chest imaging (CXR or CT) with or without positive clinical findings. $5,9,10$

Both of the incidentally diagnosed pulmonary sequestrations in our case series were left-sided and detected using the spleen as a "window" to the left lung base. Generally, trans-abdominal approach to the left lung base is more difficult than the one to the right lung base, due to anatomical relations. The liver grants complete access to the right lung base from its most anterior to its most posterior aspects, while the spleen allows the examiner to view only a small part of the left lung base, because of its lateral position, the left kidney and air from the stomach. Additionally, we do not regularly use color or power Doppler US to evaluate the spleen and the visualized region above it. Therefore, it is extremely important to know what to look for on abdominal US. The distinct gray scale US sign in both cases was an anechogenic tubular structure above the left hemidiaphragm. The use of color and power Doppler confirmed the blood flow, as well as a connection to the descending aorta. This unequivocally proved the existence of a systemic arterial supply of the solid mass within the lung base, which is one of the hallmarks of pulmonary sequestration. This finding prevents certain reported pitfalls in the diagnosis of juxtadiaphragmatic pulmonary sequestrations by US, such as missed diagnosis of neuroblastoma. ${ }^{11}$ When there is a systemic arterial supply in this part of the lung, radiologists should also consider a pseudosequestration in the differential diagnosis. In this entity, however, there is normal communication with tracheobronchial tree and normal venous drainage. Also, there is a possibility of dual arterial supply, where the part of the lung receives a supply from both systemic and pulmonary arteries. ${ }^{12}$

Our presumption, based on daily clinical work, is that sufficient attention is not necessarily paid to the pulmonary bases, as was demonstrated in the case with the 17.5-year-old girl. She had had several abdominal US examinations, none of which detected the pulmonary sequestration. The abdominal US preceding this one were performed by different pediatric radiologists.

Information about the existence of sequestration might sometimes be highly important for the clinical course and the treatment of the patient, as it was in our teenage patient, who had markedly elevated CRP and ESR. It is well known that pulmonary sequestration might represent the focus of infection. After antibiotic treatment her fever subsided, and the laboratory parameters have normalized.

In the infant girl, there were no clinical signs suggestive of sequestration. However, it is very important to know the potential etiology of the infection in the future. Further, early surgery might prevent any complications as a consequence of this congenital pulmonary malformation.

Trans-abdominal approach to both lung bases, in our experience, when the finding is normal, does not take more than 20 seconds, but it can produce very valuable information both to radiologists and clinicians, as well as enable a proper and timely diagnosis that will prevent potential complications.

In both children, we confirmed the US finding by $\mathrm{CT}$, with only a single phase of the examination performed. It was an arterial phase of contrast-enhanced CT, which precisely confirmed the US diagnosis of pulmonary sequestration, without unnecessary additional exposure to ionizing radiation. Certainly, the necessity of performing CT here is debatable. However, pediatric 
surgeons tend to require CT performed prior to any operation planning. ${ }^{6,7}$ Some of the reasons include potential hybrid lesion (congenital pulmonary airway malformation and pulmonary sequestration), detection of venous drainage, and differentiation between intralobar and extralobar types of sequestration, which is mostly based on different patterns of venous drainage. ${ }^{6,7,13}$ However, imaging differentiation between types of sequestration is often difficult, if not impossible. ${ }^{14}$ The reason is that both types of sequestration may have drainage via pulmonary veins, azygos system, or inferior vena cava. While this does not affect surgical management, the demonstration of venous anatomy may be helpful for surgical planning. ${ }^{6,7,14}$ Therefore, CT is still the diagnostic method of choice, especially for enabling an accurate operation plan, while US may be a useful diagnostic tool in the initial diagnosis and preoperative follow-up of sequestration.

\section{Conclusion}

In conclusion, pediatric abdominal US should contain a trans-abdominal examination of both lung bases. One of the potential, important, albeit rare pathological findings is pulmonary sequestration. The finding which should point towards the potential diagnosis of left-sided sequestration on gray-scale US is a tubular, sometimes branching, anechogenic structure above the left hemidiaphragm, within the consolidated area of a lung base. Finally, when the arterial blood flow is detected on CDUS within it, and a connection to the descending aorta verified, this represents an unequivocal sign of pulmonary sequestration.

\section{References}

1. Vijayaraghavan SB, Rao PS, Selvarasu CD, Rao TM. Prenatal sonographic features of intralobar bronchopulmonary sequestration. J Ultrasound Med 2003;22:541-4.
2. Biyyam DR, Chapman T, Ferguson MR, Deutsch G, Dighe MK. Congenital lung abnormalities: embryologic features, prenatal diagnosis, and postnatal radiologic-pathologic correlation. Radiographics 2010;30:1721-38.

3. Ruano R, Benachi A, Aubry MC, Revillon Y, Emond S, Dumez Y, et al. Prenatal diagnosis of pulmonary sequestration using three-dimensional power Doppler ultrasound. Ultrasound Obstet Gynecol 2005;25:128-33.

4. Sepulveda W. Perinatal imaging in bronchopulmonary sequestration. J Ultrasound Med 2009;28:89-94.

5. West MS, Donaldson JS, Shkolnik A. Pulmonary sequestration. Diagnosis by ultrasound. J Ultrasound Med 1989;8:125-9.

6. Lee EY, Siegel MJ, Sierra LM, Foglia RP. Evaluation of angioarchitecture of pulmonary sequestration in pediatric patients using 3D MDCT angiography. AJR Am J Roentgenol 2004; 183:183-8.

7. Abbey P, Das CJ, Pangtey GS, Seith A, Dutta R, Kumar A. Imaging in bronchopulmonary sequestration. J Med Imaging Radiat Oncol 2009;53:22-31.

8. Adaletli I, Uludag S. Intraabdominal extrapulmonary sequestration diagnosed by color doppler ultrasound at 22 weeks' gestation. J Clin Ultrasound 2013;41:251-4.

9. Schlesinger AE, DiPietro MA, Statter MB, Lally KP. Utility of sonography in the diagnosis of bronchopulmonary sequestration. J Pediatr Surg 1994;29:52-5.

10. Thind CR, Pilling DW. Pulmonary sequestration: the value of ultrasound. Clin Radiol 1985;36:437-9.

11. Manson DE, Daneman A. Pitfalls in the sonographic diagnosis of juxtadiaphragmatic pulmonary sequestrations. Pediatr Radiol 2001;31:260-4.

12. Irodi A, Prabhu SM, John RA, Leena RV. Congenital bronchopulmonary vascular malformations, "sequestration" and beyond. Indian J Radiol Imaging 2015;25:35-43.

13. Samuel M, Burge DM. Management of antenatally diagnosed pulmonary sequestration associated with congenital cystic adenomatoid malformation. Thorax 1999;54:701-6.

14. Donnelly LF. Pulmonary sequestration. In: Donnelly LF, editor. Diagnostic imaging: pediatrics. Salt Lake City: Amirsys; 2005; p.10-3. 